\title{
INTERPOLATING WITH OUTER FUNCTIONS
}

\author{
JAVAD MASHREGHI, MAREK PTAK, AND WILLIAM T. ROSS
}

\begin{abstract}
The classical theorems of Mittag-Leffler and Weierstrass show that when $\left\{\lambda_{n}\right\}$ is a sequence of distinct points in the open unit disk $\mathbb{D}$, with no accumulation points in $\mathbb{D}$, and $\left\{w_{n}\right\}$ is any sequence of complex numbers, there is an analytic function $\varphi$ on $\mathbb{D}$ for which $\varphi\left(\lambda_{n}\right)=w_{n}$. A celebrated theorem of Carleson [2] characterizes when, for a bounded sequence $\left\{w_{n}\right\}$, this interpolating problem can be solved with a bounded analytic function. A theorem of Earl [7] goes further and shows that when Carleson's condition is satisfied, the interpolating function $\varphi$ can be a constant multiple of a Blaschke product. In this paper, we explore when the interpolating $\varphi$ can be an outer function. We then use our results to refine a result of McCarthy 12 and explore the common range of the co-analytic Toeplitz operators on a model space.
\end{abstract}

\section{INTERPOLATION}

Interpolation problems for analytic functions have been a mainstay in complex analysis since its conception in the late 19th century. The general idea is that we have a certain class $\mathcal{X}$ of analytic functions on the open unit disk $\mathbb{D}$ (e.g., all analytic functions, bounded analytic functions, analytic self maps of $\mathbb{D}$, Blaschke products, outer functions). Then, for a sequence $\left\{\lambda_{n}\right\}$ of distinct points in $\mathbb{D}$ and sequence $\left\{w_{n}\right\}$ of complex numbers, we want to find an $f \in \mathcal{X}$ such that $f\left(\lambda_{n}\right)=w_{n}$ for all $n$. If we are not able to solve this problem for all $\left\{\lambda_{n}\right\}$ and $\left\{w_{n}\right\}$, what restrictions must we have?

Suppose $\mathcal{X}$ is the class of all analytic functions on $\mathbb{D}$. For a sequence $\left\{\lambda_{n}\right\}$ of distinct points in $\mathbb{D}$ (with no limit point in $\mathbb{D}$ ) and any sequence $\left\{w_{n}\right\}$, an application of the classical Mittag-Leffler theorem and the Weierstrass factorization theorem produces an analytic function $f$ with $f\left(\lambda_{n}\right)=w_{n}$ for all $n$. In other words, for the class $\mathcal{X}$ of all

2010 Mathematics Subject Classification. 30H10, 47B35, 30E05, 41A05.

Key words and phrases. Interpolating sequences, Hardy spaces, outer functions, Toeplitz operators, model spaces.

This work was supported by the NSERC Discovery Grant (Canada) and by the Ministry of Science and Higher Education of the Republic of Poland. 
analytic functions on $\mathbb{D}$, besides the obvious restriction that $\left\{\lambda_{n}\right\}$ has no limit points in $\mathbb{D}$, there is no other restriction on $\left\{\lambda_{n}\right\}$ to be able to interpolate any sequence $\left\{w_{n}\right\}$ with an analytic function.

Of course, there are the finite interpolation problems. For example, a well known result of Lagrange, from 1795, says that given distinct $\lambda_{1}, \ldots, \lambda_{n}$ in $\mathbb{C}$ and arbitrary $w_{1}, \ldots, w_{n}$ in $\mathbb{C}$ there is a polynomial $p$ of degree $n-1$ such that $p\left(\lambda_{j}\right)=w_{j}$ for all $1 \leqslant j \leqslant n$. There is also the often-quoted result of Nevanlinna and Pick (from 1916) which says that given distinct $\lambda_{1}, \ldots, \lambda_{n}$ in $\mathbb{D}$ and arbitrary $w_{1}, \ldots, w_{n}$ in $\mathbb{D}$, there is an $f \in H^{\infty}$ with $|f| \leqslant 1$ on $\mathbb{D}$ for which $f\left(\lambda_{j}\right)=w_{j}, 1 \leqslant j \leqslant n$, if and only if the Nevanlinna-Pick matrix

$$
\left[\frac{1-\overline{w_{j}} w_{i}}{1-\overline{\lambda_{j}} \lambda_{i}}\right]_{1 \leqslant i, j \leqslant n}
$$

is positive semidefinite [1, 9].

When $\mathcal{X}$ is the class of bounded analytic functions on $\mathbb{D}$, denoted in the literature by $H^{\infty}$, a well-known theorem of Carleson [2] (see also [9]) says the following: A sequence $\Lambda=\left\{\lambda_{n}\right\} \subseteq \mathbb{D}$ has the property that given any bounded sequence $\left\{w_{n}\right\}$ there is a $\varphi \in H^{\infty}$ such that $\varphi\left(\lambda_{n}\right)=w_{n}$ if and only if

$$
\delta(\Lambda):=\inf _{n \geqslant 1} \prod_{k=1, k \neq n}^{\infty}\left|\frac{\lambda_{k}-\lambda_{n}}{1-\bar{\lambda}_{k} \lambda_{n}}\right|>0 .
$$

Such $\left\{\lambda_{n}\right\}$ are called interpolating sequences. In this paper we explore the type of functions $\varphi \in H^{\infty}$ that can perform the interpolating. For example, a result of Earl [7] says that when $\delta(\Lambda)>0$ one can always take the interpolating function $\varphi$ to be a constant multiple of a Blaschke product. Other types of interpolation problems are discussed in [6, 13].

Inspired by a common range problem for co-analytic Toeplitz operators on model spaces that we will discuss at the end of this paper, we focus on conditions on the targets $\left\{w_{n}\right\}$ that allow us to take $\varphi$ to be an outer function (bounded outer function). Our results are as follows: In Theorem 3.1 we prove that for interpolating $\left\{\lambda_{n}\right\}$ and bounded $\left\{w_{n}\right\}$ with

$$
\inf _{n \geqslant 1}\left|w_{n}\right|>0,
$$

there is a bounded outer function $\varphi$ such that $\varphi\left(\lambda_{n}\right)=w_{n}$ for all $n$. As an application of this, we prove in Proposition 3.6 that when $\left\{w_{n}\right\}$ 
and $\left\{w_{n}^{\prime}\right\}$ are bounded with

$$
0<m \leqslant\left|\frac{w_{n}}{w_{n}^{\prime}}\right| \leqslant M<\infty, \quad n \geqslant 1
$$

then $\left\{w_{n}\right\}$ can be interpolated by an outer function (bounded outer function) if and only if $\left\{w_{n}^{\prime}\right\}$ can as well. Therefore, for subsequent discussions, without loss of generality, we may consider positive target sequences. To establish conditions for which $\left\{w_{n}\right\}$ can be interpolated by an outer function, we prove in Theorem 4.1 that when $\left\{w_{n}\right\} \subseteq$ $\mathbb{C} \backslash\{0\}$ can be interpolated by an outer function it must be the case that

$$
\lim _{n \rightarrow \infty}\left(1-\left|\lambda_{n}\right|\right) \log \left|w_{n}\right|=0 .
$$

Hence sequences such as

$$
w_{n}=e^{-\frac{1}{1-\left|\lambda_{n}\right|}}, \quad n \geqslant 1
$$

can not be interpolated by an outer function. In other words, any $H^{\infty}$ function $\varphi$ for which $\varphi\left(\lambda_{n}\right)=w_{n}$ for all $n$ (and such $\varphi$ exist by Carleson's theorem) must have an inner factor. In fact (Theorem 5.1), any bounded analytic function $\varphi$ which satisfies the stronger decay condition

$$
\varphi\left(\lambda_{n}\right)=e^{-\frac{1}{\left(1-\left|\lambda_{n}\right|\right)^{2}}}
$$

must have a Blaschke factor. In Theorem 6.10 and Theorem 7.5 we discuss the sharpness of Theorem 4.1 and explore conditions on the decay the rate of $\left(1-\left|\lambda_{n}\right|\right) \log \left|w_{n}\right|$ to determine when there is an outer function (bounded outer function) that interpolates $\left\{w_{n}\right\}$.

Worth mentioning here is the paper [6] which examines the question of when the interpolating function can be zero free. The outer functions are a strict subclass of the zero free functions since the zero free functions can have a singular inner factor.

In the final part of this paper we apply our results to determine the common range for the co-analytic Toeplitz operators on a model space. In fact, this was our original reason for exploring this topic. For an inner function $u$, define the model space $\mathcal{K}_{u}=\left(u H^{2}\right)^{\perp}$. It is known [8, p. 106] that $\mathcal{K}_{u}$ is an invariant subspace for any co-analytic Toeplitz operator $T_{\bar{\varphi}}$ on $H^{2}$. In [12] McCarthy described the set

$$
\mathscr{R}\left(H^{2}\right):=\bigcap\left\{T_{\bar{\varphi}} H^{2}: \varphi \in H^{\infty} \backslash\{0\}\right\}
$$


the functions in the common range of all the (nonzero) co-analytic Toeplitz operators. By the Douglas factorization theorem (see $\$ 8$ below), $H^{\infty} \backslash\{0\}$ can be replaced by $H^{\infty} \cap \mathcal{O}$, where $\mathcal{O}$ is the class of outer functions.

As an application of our outer interpolation results, we determine

$$
\mathscr{R}\left(\mathcal{K}_{u}\right):=\bigcap\left\{T_{\bar{\varphi}} \mathcal{K}_{u}: \varphi \in \mathcal{O} \cap H^{\infty}\right\}
$$

the common range on a fixed model space. While $\mathscr{R}\left(H^{2}\right)$ is somehow "large", for example, it contains all functions that are analytic in a neighborhood of $\overline{\mathbb{D}}, \mathscr{R}\left(\mathcal{K}_{u}\right)$ can be considerably smaller. In fact $\mathscr{R}\left(\mathcal{K}_{u}\right)=\{0\}$ for certain $u$ (Example 8.6). We describe $\mathscr{R}\left(\mathcal{K}_{u}\right)$ for any inner function (Theorem 8.7) and when $u$ is an interpolating Blaschke product (zeros are an interpolating sequence), we give an alternate, and more tangible, description involving our outer interpolating results (Theorem 9.4).

\section{Some notation}

Let us set our notation and review some well-known facts about the classes of analytic functions that appear in this paper. The books [4, 9] are thorough references for the details and proofs. In this paper, $\mathbb{D}$ is the open unit disk $\{z \in \mathbb{C}:|z|<1\}, \mathbb{T}$ the unit circle $\{z \in \mathbb{C}:|z|=1\}$, and $d m=d \theta / 2 \pi$ is normalized Lebesgue measure on $\mathbb{T}$. The space $H^{1}$, the Hardy space, is the set of analytic functions $f$ on $\mathbb{D}$ for which

$$
\sup _{0<r<1} \int_{\mathbb{T}}|f(r \xi)| d m(\xi)<\infty .
$$

Standard results say that every $f \in H^{1}$ has a radial limit

$$
f(\xi):=\lim _{r \rightarrow 1^{-}} f(r \xi)
$$

for almost every $\xi \in \mathbb{T}$ and

$$
\int_{\mathbb{T}}|f(\xi)| d m(\xi)=\sup _{0<r<1} \int_{\mathbb{T}}|f(r \xi)| d m(\xi) .
$$

As is the usual practice in Hardy spaces, we use the symbol $f$ to denote the boundary function on $\mathbb{T}$ as well as the analytic function on $\mathbb{D}$.

We let $H^{\infty}$ be the bounded analytic functions on $\mathbb{D}$ and observe that $H^{\infty} \subseteq H^{1}$ and thus every $f \in H^{\infty}$ also has a radial boundary function. In fact,

$$
\sup _{z \in \mathbb{D}}|f(z)|=\operatorname{ess-sup}_{\mathbb{T}}|f|
$$


If $W$ is an extended real-valued integrable function on $\mathbb{T}$,

$$
\varphi(z)=\exp \left(\int_{\mathbb{T}} \frac{\xi+z}{\xi-z} W(\xi) d m(\xi)\right), \quad z \in \mathbb{D},
$$

is analytic on $\mathbb{D}$ and is called an outer function. Observe that

$$
\log |\varphi(z)|=\int_{\mathbb{T}} \Re\left(\frac{\xi+z}{\xi-z}\right) W(\xi) d m(\xi)=\int_{\mathbb{T}} \frac{1-|z|^{2}}{|\xi-z|^{2}} W(\xi) d m(\xi),
$$

which is the Poisson integral of $W$. By some harmonic analysis [9, p. 15],

$$
\lim _{r \rightarrow 1^{-}} \log |\varphi(r \zeta)|=\log |\varphi(\zeta)|=W(\zeta)
$$

for almost every $\zeta \in \mathbb{T}$. This process can be reversed and so given an extended real-valued $W \in L^{1}(\mathbb{T})$, there is an outer $\varphi$ with

$$
|\varphi(\zeta)|=e^{W(\zeta)}
$$

almost everywhere (in terms of radial boundary values).

The outer functions belong to the Smirnov class

$$
N^{+}=\left\{f / g: f \in H^{\infty}, g \in H^{\infty} \cap \mathcal{O}\right\}
$$

and every $F \in N^{+}$can be factored as $F=I_{F} O_{F}$, where $I_{F}$ is inner $\left(I_{F} \in H^{\infty}\right.$ with unimodular boundary values almost everywhere on $\mathbb{T}$ ) and $O_{F}$ is outer. There are also the inclusions $H^{\infty} \subseteq H^{1} \subseteq N^{+}$.

\section{Positive Results}

We start off with examples of bounded $\left\{w_{n}\right\}$ which can be interpolated by outer functions and explore the ones which can not in the next section.

Theorem 3.1. Suppose $\left\{\lambda_{n}\right\} \subseteq \mathbb{D}$ is interpolating. For a bounded $\left\{w_{n}\right\}$ with

$$
\inf _{n \geqslant 1}\left|w_{n}\right|>0
$$

there is a bounded outer function $\varphi$ such that $\varphi\left(\lambda_{n}\right)=w_{n}$ for all $n$.

The proof requires a few preliminaries. The first is a more detailed version of Carleson's result on interpolating sequences [11, p. 268].

Theorem 3.2 (Carleson). For an interpolating $\Lambda=\left\{\lambda_{n}\right\}$ there is a constant $0<C(\Lambda)<1$ with the following properties.

(i) For each $\left\{w_{n}\right\}$ satisfying $\left|w_{n}\right| \leqslant C(\Lambda)$ for all $n \geqslant 1$, there is an $\varphi \in H^{\infty}$ such that $\|\varphi\|_{\infty} \leqslant 1$ and $\varphi\left(\lambda_{n}\right)=w_{n}$ for all $n$. 
(ii) There are bounded $\left\{w_{n}\right\}$ with $\left|w_{n}\right|>C(\Lambda)$, for at least one $n$, such that $\|\varphi\|_{\infty}>1$ for any interpolating function $\varphi \in H^{\infty}$.

The number $C(\Lambda)$ is called the Carleson index for $\Lambda$ and is related to $\delta(\Lambda)$ from (1.1) by

$$
A \frac{\delta(\Lambda)}{\log \left(\frac{e}{\delta(\Lambda)}\right)} \leqslant C(\lambda) \leqslant \delta(\Lambda)
$$

where $A$ is an absolute constant [11, p. 268]. Our first step is to prove a special case of Theorem 3.1 .

Lemma 3.3. Suppose $\left\{w_{n}\right\} \subseteq \overline{D(a, r)}=\{z:|z-a| \leqslant r\}$. If $r /|a|<$ $C(\Lambda)$ there is a bounded outer $\varphi$ such that $\varphi\left(\lambda_{n}\right)=w_{n}$ for all $n$.

The hypothesis $r /|a|<C(\Lambda)$ implies that $r<|a|$ and so $\overline{D(a, r)}$ does not contain the origin.

Proof. Set

$$
t_{n}:=C(\Lambda) \frac{w_{n}-a}{r} .
$$

Then $\left|t_{n}\right| \leqslant C(\Lambda)$, and, by Theorem 3.2, there is $g \in H^{\infty}$ with $\|g\|_{\infty} \leqslant$ 1 and such that $g\left(\lambda_{n}\right)=t_{n}$ for all $n$. Define

$$
\varphi:=\frac{r}{C(\Lambda)} g+a
$$

Clearly $\varphi \in H^{\infty}$ and

$$
\varphi\left(\lambda_{n}\right)=\frac{r}{C(\Lambda)} g\left(\lambda_{n}\right)+a=\frac{r}{C(\Lambda)} t_{n}+a=w_{n} .
$$

Moreover, with $a=|a| e^{i \alpha}$ and $z \in \mathbb{D}$,

$$
\Re\left(e^{-i \alpha} \varphi(z)\right)=\frac{r}{C(\Lambda)} \Re\left(e^{-i \alpha} g(z)\right)+|a| \geqslant \frac{r}{C(\Lambda)}\left(\Re\left(e^{-i \alpha} g(z)\right)+1\right)
$$

which is positive. The condition $\Re\left(e^{-i \alpha} \varphi\right)>0$ is sufficient to ensure that $e^{-i \alpha} \varphi$ is an outer function [9, p. 65]. Thus $\varphi$ is outer as well.

Remark 3.4. When $\left\{w_{n}\right\} \subseteq(0, \infty)$, we can choose $\alpha=0$ in the above proof and thus choose the interpolating function to satisfy $\Re \varphi>0$. This detail will become important later on. 
Remark 3.5. If one just wanted a nonvanishing interpolating function $\varphi \in H^{\infty}$ in Theorem 3.1, one could take $\varphi=e^{\psi}$, where $\psi \in H^{\infty}$ with $\psi\left(\lambda_{n}\right)=\log w_{n}$ (for a suitably defined logarithm). See [6] for more on this.

Proof of Theorem 3.2. Fix $0<r<C(\Lambda)$ and consider the closed disk $\overline{D(1, r)}$. Since $m \leqslant\left|w_{n}\right| \leqslant M, n \geqslant 1$, for some positive constants $m$ and $M$, there is a positive integer $k$ such that $w_{n}^{1 / k} \in \overline{D(1, r)}$ for all $n \geqslant 1$. We use the main branch of logarithm to evaluate $w_{n}^{1 / k}$. Therefore, by Lemma 3.3, there is a bounded outer function $g$ such that $g\left(\lambda_{n}\right)=w_{n}^{1 / k}$ for all $n$. The function $\varphi:=g^{k}$ is bounded, outer, and $\varphi\left(\lambda_{n}\right)=w_{n}$ for all $n$.

This next result says that for outer interpolation, we can always assume, for example, that the targets $w_{n}$ are positive.

Proposition 3.6. Suppose $\left\{\lambda_{n}\right\}$ is interpolating and $\left\{w_{n}\right\}$ and $\left\{w_{n}^{\prime}\right\}$ are bounded with

$$
0<m \leqslant\left|\frac{w_{n}^{\prime}}{w_{n}}\right| \leqslant M<\infty, \quad n \geqslant 1
$$

Then $\left\{w_{n}\right\}$ can be interpolated by an outer (bounded outer) function if and only if $\left\{w_{n}^{\prime}\right\}$ can be interpolated by an outer (bounded outer) function.

Proof. By Theorem 3.1 there is a bounded outer $F$ such that $F\left(\lambda_{n}\right)=$ $w_{n}^{\prime} / w_{n}$ for all $n \geqslant 1$. If there is an outer (bounded outer) $\varphi$ such that $\varphi\left(\lambda_{n}\right)=w_{n}$ for all $n$, then the outer (bounded outer) $\varphi F$ performs the desired interpolation for $\left\{w_{n}^{\prime}\right\}$.

Remark 3.7. If $\varphi$ is outer (bounded outer) then so is $\varphi^{c}$ for any $c>0$. Thus $\left\{w_{n}^{c}\right\}$ can be interpolated by an outer (bounded outer) function whenever $\left\{w_{n}\right\}$ can.

\section{Negative RESUlts - EXISTENCE OF AN INNER FACTOR}

If $\left\{\lambda_{n}\right\}$ is interpolating we know that given any bounded $\left\{w_{n}\right\}$ there is a $\varphi \in H^{\infty}$ such that $\varphi\left(\lambda_{n}\right)=w_{n}$. This next result says that under certain circumstances, any Smirnov interpolating function for $\left\{w_{n}\right\}$ must have an inner factor. 
Theorem 4.1. If $\left\{\lambda_{n}\right\}$ is interpolating and $\left\{w_{n}\right\} \subseteq \mathbb{C} \backslash\{0\}$ satisfies

$$
\lim _{n \rightarrow \infty}\left(1-\left|\lambda_{n}\right|\right) \log \left|w_{n}\right| \neq 0,
$$

then any $\varphi \in N^{+}$satisfying $\varphi\left(\lambda_{n}\right)=w_{n}$ for all $n$ must have a nontrivial inner factor.

\section{Example 4.2. If}

$$
w_{n}:=e^{-\frac{1}{1-\left|\lambda_{n}\right|}}, \quad n \geqslant 1,
$$

then any interpolating $\varphi \in N^{+}$for $\left\{w_{n}\right\}$ is not outer.

This result will follow from the lemma below which is probably folklore but we include a proof for completeness.

Lemma 4.3. If $\varphi$ is outer, then

$$
\lim _{|z| \rightarrow 1^{-}}(1-|z|) \log |\varphi(z)|=0 \text {. }
$$

Proof. Let $a>1$ and $E_{a}=\{\xi \in \mathbb{T}:|\varphi(\xi)|>a\}$. Then $\log |\varphi|>0$ on $E_{a}$ and an application of

$$
\int_{\mathbb{T}} \frac{1-|z|^{2}}{|\xi-z|^{2}} d m(\xi)=1, \quad z \in \mathbb{D} ;
$$

and

$$
\frac{1-|z|^{2}}{|\xi-z|^{2}} \leqslant \frac{2}{1-|z|}, \quad z \in \mathbb{D}, \quad \xi \in \mathbb{T} .
$$

give us

$$
\begin{aligned}
\log |\varphi(z)| & =\int_{\mathbb{T}} \frac{1-|z|^{2}}{|z-\xi|^{2}} \log |\varphi(\xi)| d m(\xi) \\
& =\int_{E_{a}} \frac{1-|z|^{2}}{|z-\xi|^{2}} \log |\varphi(\xi)| d m(\xi)+\int_{\mathbb{T} \backslash E_{a}} \frac{1-|z|^{2}}{|z-\xi|^{2}} \log |\varphi(\xi)| d m(\xi) \\
& \leqslant \frac{2}{1-|z|} \int_{E_{a}} \log |\varphi(\xi)| d m(\xi)+\log a \int_{\mathbb{T} \backslash E_{a}} \frac{1-|z|^{2}}{|z-\xi|^{2}} d m(\xi) \\
& \leqslant \frac{2}{1-|z|} \int_{E_{a}} \log |\varphi(\xi)| d m(\xi)+\log a .
\end{aligned}
$$

Hence,

$$
(1-|z|) \log |\varphi(z)| \leqslant 2 \int_{E_{a}} \log |\varphi(\xi)| d m(\xi)+(1-|z|) \log a, \quad z \in \mathbb{D}
$$


which implies

$$
\varlimsup_{|z| \rightarrow 1^{-}}(1-|z|) \log |\varphi(z)| \leqslant 2 \int_{E_{a}} \log |\varphi(\xi)| d m(\xi) .
$$

Now let $a \rightarrow+\infty$ and use the fact that $\log |\varphi| \in L^{1}(\mathbb{T})$ to deduce

$$
\varlimsup_{|z| \rightarrow 1^{-}}(1-|z|) \log |\varphi(z)| \leqslant 0 .
$$

Since $1 / \varphi$ is also outer, the above argument implies

$$
\varlimsup_{|z| \rightarrow 1^{-}}(1-|z|) \log |1 / \varphi(z)| \leqslant 0
$$

or equivalently

$$
\underline{|z| \rightarrow 1^{-}}(1-|z|) \log |\varphi(z)| \geqslant 0 .
$$

The result now follows by comparing (4.6) and (4.7).

Let us comment here that when the hypothesis of Theorem 4.1 is satisfied, the inner factor that appears in the interpolating function $\varphi$ plays a significant role in the decay of $\varphi$.

Corollary 4.8. Suppose $\left\{\lambda_{n}\right\}$ is interpolating and $\left\{w_{n}\right\} \subseteq \mathbb{C} \backslash\{0\}$ is a bounded and satisfies

$$
\lim _{n \rightarrow \infty}\left(1-\left|\lambda_{n}\right|\right) \log \left|w_{n}\right| \neq 0 .
$$

If $I_{\varphi}$ is the inner factor for a $\varphi \in N^{+}$for which $\varphi\left(\lambda_{n}\right)=w_{n}$ for all $n$, then

$$
\varliminf_{n \rightarrow \infty}\left|I_{\varphi}\left(\lambda_{n}\right)\right|=0
$$

Proof. Let $\varphi=F_{\varphi} I_{\varphi}$, where $F_{\varphi}$ is outer and $I_{\varphi}$ is inner. If $\left|I_{\varphi}\left(\lambda_{n}\right)\right| \geqslant$ $\delta>0$ for all $n$, then $I_{\varphi}\left(\lambda_{n}\right)=w_{n} / F_{\varphi}\left(\lambda_{n}\right)$ satisfies the hypothesis of Theorem 3.1 and so there is a bounded outer $\psi$ with $\psi\left(\lambda_{n}\right)=I_{\varphi}\left(\lambda_{n}\right)$ and so $F_{\varphi} \psi$ is outer and interpolates $w_{n}$. This says that $w_{n}$ can be interpolated by an outer function - which it can not.

Remark 4.9. The above says that a subsequence of $\left\{\lambda_{n}\right\}$ must approach

$$
\left\{\xi \in \mathbb{T}: \underline{\lim }_{z \rightarrow \xi}\left|I_{\varphi}(z)\right|=0\right\},
$$

the boundary spectrum of the inner factor $I_{\varphi}$. This set will consist of the accumulation of the zeros of the Blaschke factor of $I_{\varphi}$ as well as 
the support of the singular measure associated with the singular inner inner factor of $I_{\varphi}[8$, p. 152].

\section{Negative Results - existence of a Blaschke product}

This next result says that under the right circumstances, any Smirnov interpolating must have a Blaschke factor.

Theorem 5.1. Suppose $\left\{\lambda_{n}\right\}$ is interpolating and $\left\{w_{n}\right\} \subseteq \mathbb{C} \backslash\{0\}$ is bounded and satisfies

$$
\varlimsup_{n \rightarrow \infty}\left(1-\left|\lambda_{n}\right|\right)|\log | w_{n}||=\infty .
$$

Then any $\varphi \in N^{+}$for which $\varphi\left(\lambda_{n}\right)=w_{n}$ for all $n \geqslant 1$ must have a Blaschke factor.

This says, for example, that for an interpolating $\left\{\lambda_{n}\right\}$ any $\varphi \in H^{\infty}$ for which

$$
\varphi\left(\lambda_{n}\right)=\exp \left(-\frac{1}{\left(1-\left|\lambda_{n}\right|\right)^{2}}\right)
$$

(and such $\varphi$ exist by Carleson's theorem) must have a Blaschke factor.

The proof of this theorem follows from the following lemma on zero-free Smirnov functions. Any zero-free $\varphi \in N^{+}$can be written as

$$
\varphi(z)=\exp \left(\int_{\mathbb{T}} \frac{\xi+z}{\xi-z} W(\xi) d m(\xi)\right) \exp \left(-\int_{\mathbb{T}} \frac{\xi+z}{\xi-z} d \mu(\xi)\right),
$$

where $W$ is a real-valued integrable function and $\mu$ is a positive measure that is singular with respect to Lebesgue measure $m$.

Lemma 5.3. If $\varphi \in N^{+}$and zero free, then

$$
\varlimsup_{|z| \rightarrow 1^{-}}(1-|z|)|\log | \varphi(z)||<\infty .
$$

Proof. From (5.2) we have

$$
\log |\varphi(z)|=\int_{\mathbb{T}} \frac{1-|z|^{2}}{|z-\xi|^{2}} W(\xi) d m(\xi)-\int_{\mathbb{T}} \frac{1-|z|^{2}}{|z-\xi|^{2}} d \mu(\xi) .
$$

The proof of Lemma 4.3 shows that

$$
\lim _{|z| \rightarrow 1^{-}}(1-|z|) \int_{\mathbb{T}} \frac{1-|z|^{2}}{|z-\xi|^{2}} W(\xi) d m(\xi)=0 .
$$

From (4.5) we have

$$
0 \leqslant(1-|z|) \int_{\mathbb{T}} \frac{1-|z|^{2}}{|z-\xi|^{2}} d \mu(\xi) \leqslant 2 \int_{\mathbb{T}} d \mu=2 \mu(\mathbb{T}) .
$$


Combine these two facts to prove the result.

\section{A GRowth RATE CHARACTERIZATION}

We know from Corollary 4.8 that if $\left\{w_{n}\right\}$ can be interpolated by an outer function, then

$$
\lim _{n \rightarrow \infty}\left(1-\lambda_{n}\right) \log \left|w_{n}\right|=0
$$

What is the decay rate of $\left(1-\lambda_{n}\right) \log \left|w_{n}\right|$ ? Here we focus our attention on the case when $\left\{\lambda_{n}\right\} \subseteq(0,1)$. Though it does not play a role in our results, it is known [4, p. 156] that $\left\{\lambda_{n}\right\} \subseteq(0,1)$ is interpolating if and only if there is a $0<c<1$ such that

$$
\left(1-\lambda_{n+1}\right) \leqslant c\left(1-\lambda_{n}\right), \quad n \geqslant 1 .
$$

Such sequences are called exponential sequences. Naively speaking, the following result says that the decay rate of $\left(1-\lambda_{n}\right) \log \left|w_{n}\right|$ is controlled by an absolutely continuous function. The sharpness of this observation will be studied in Theorem 6.10.

Theorem 6.1. Suppose $\left\{\lambda_{n}\right\} \subseteq(0,1)$ is interpolating and $\left\{w_{n}\right\} \subseteq$ $\mathbb{C} \backslash\{0\}$ is a bounded with

$$
M:=\sup _{n \geqslant 1}\left|w_{n}\right| .
$$

Suppose there is an outer function $\varphi$ for which $\varphi\left(\lambda_{n}\right)=w_{n}$ for all $n$. Then there is a positive, decreasing, integrable function $h$ on $[0,1]$ such that

$$
-\left(1-\lambda_{n}\right) \log \left|\frac{w_{n}}{M}\right| \leqslant \int_{0}^{1-\lambda_{n}} h(t) d t, \quad n \geqslant 1 .
$$

To prepare for the proof, we require a few comments on the growth of the Poisson kernel. We begin with the following two normalizing assumptions on $h$ :

$$
h \text { is right continuous; }
$$

$$
h \text { is extended to }[0, \pi] \text { by setting } h(x)=0 \text { for } x \in[1, \pi] .
$$

The right continuity can be assumed since $h$ is monotone and thus has at most a countable number of jumps. Since the behavior around the origin is our main concern, extending the definition of $h$ to $[0, \pi]$ is merely for aesthetic purposes when working with Poisson integrals below. Let

$$
P_{r}(t)=\frac{1-r^{2}}{1-2 r \cos t+r^{2}}, \quad 0 \leqslant r<1, \quad-\pi \leqslant t \leqslant \pi,
$$


be the standard Poisson kernel. We wish to examine the function

$$
A_{h}(r)=(1-r) \int_{-\pi}^{\pi} P_{r}(t) h(|t|) \frac{d t}{2 \pi} .
$$

For $r \in[0,1)$ and $t \in[-\pi, \pi]$ we have

$$
\frac{(1-r)\left(1-r^{2}\right)}{1-2 r \cos t+r^{2}}=\frac{(1+r)(1-r)^{2}}{(1-r)^{2}+4 r \sin ^{2}(t / 2)}
$$

This yields

$$
\begin{aligned}
A_{h}(r) & =\int_{-\pi}^{\pi} \frac{(1-r)\left(1-r^{2}\right)}{1-2 r \cos t+r^{2}} h(|t|) \frac{d t}{2 \pi} \\
& =2 \int_{0}^{\pi} \frac{(1+r)(1-r)^{2}}{(1-r)^{2}+4 r \sin ^{2}(t / 2)} h(t) \frac{d t}{2 \pi} \\
& \geqslant 2 \int_{0}^{1-r} \frac{(1+r)(1-r)^{2}}{(1-r)^{2}+4 r \sin ^{2}(t / 2)} h(t) \frac{d t}{2 \pi}
\end{aligned}
$$

Note that

$$
t^{2} \sin ^{2}\left(\frac{1}{2}\right) \leqslant \sin ^{2}\left(\frac{t}{2}\right) \leqslant \frac{1}{4} t^{2}
$$

and so

$$
\begin{aligned}
\frac{1}{\pi} \int_{0}^{1-r} \frac{(1+r)(1-r)^{2}}{(1-r)^{2}+4 \sin ^{2}(t / 2)} h(t) d t & \geqslant \frac{1}{\pi} \int_{0}^{1-r} \frac{(1-r)^{2}}{(1-r)^{2}+t^{2}} h(t) d t \\
& \geqslant \frac{1}{\pi} \int_{0}^{1-r} \frac{(1-r)^{2}}{(1-r)^{2}+(1-r)^{2}} h(t) d t \\
& =\frac{1}{2 \pi} \int_{0}^{1-r} h(t) d t .
\end{aligned}
$$

In summary,

$$
A_{h}(r) \geqslant \frac{1}{2 \pi} \int_{0}^{1-r} h(t) d t
$$

To obtain an upper bound, note that $A(r)$ is equal to the sum of

$$
\frac{1}{\pi} \int_{0}^{1-r} \frac{(1-r)\left(1-r^{2}\right)}{(1-r)^{2}+4 r \sin ^{2}(t / 2)} h(t) d t
$$

and

$$
\frac{1}{\pi} \int_{1-r}^{\pi} \frac{(1-r)\left(1-r^{2}\right)}{1-2 r \cos t+r^{2}} h(t) d t
$$

For the first integral, observe that

$$
\frac{1}{\pi} \int_{0}^{1-r} \frac{(1+r)(1-r)^{2}}{(1-r)^{2}+4 r \sin ^{2}(t / 2)} h(t) d t \leqslant \frac{1}{\pi} \int_{0}^{1-r} \frac{(1+r)(1-r)^{2}}{(1-r)^{2}} h(t) d t
$$




$$
\leqslant \frac{2}{\pi} \int_{0}^{1-r} h(t) d t
$$

For the second integral we recall from (6.3) that $h(\pi)=0$ and we use the second mean value theorem [14 for integrals, along with the right continuity of $h$ from (6.2), to see that

$$
\frac{1}{\pi} \int_{1-r}^{\pi} \frac{(1-r)\left(1-r^{2}\right)}{1-2 r \cos t+r^{2}} h(t) d t=\frac{1}{\pi} h(1-r) \int_{1-r}^{t_{0}} \frac{(1-r)\left(1-r^{2}\right)}{1-2 r \cos t+r^{2}} d t
$$

for some $t_{0} \in[1-r, \pi]$. Notice that

$$
\begin{aligned}
\frac{1}{\pi} h(1-r) \int_{1-r}^{t_{0}} \frac{(1-r)\left(1-r^{2}\right)}{1-2 r \cos t+r^{2}} d t & =\frac{1}{\pi}(1-r) h(1-r) \int_{1-r}^{t_{0}} P_{r}(t) d t \\
& \leqslant(1-r) h(1-r) .
\end{aligned}
$$

In the last step note the use of

$$
\int_{1-r}^{t_{0}} P_{r}(t) d t \leqslant \int_{0}^{\pi} P_{r}(t) d t=\pi
$$

We now use the fact that $h$ is decreasing to obtain

$$
\int_{0}^{1-r} h(t) d t \geqslant h(1-r) \int_{0}^{1-r} d t=(1-r) h(1-r) .
$$

Put this all together to get

$$
A_{h}(r) \leqslant \frac{2+\pi}{\pi} \int_{0}^{1-r} h(t) d t .
$$

Thus, combining (6.6) and (6.7) we have the summary estimate

$$
\frac{1}{2 \pi} \int_{0}^{1-r} h(t) d t \leqslant A_{h}(r) \leqslant \frac{2+\pi}{\pi} \int_{0}^{1-r} h(t) d t .
$$

An important tool for our next step is the symmetric decreasing rearrangement. If $E$ is a measurable subset of $[-\pi, \pi]$ let

$$
E^{*}=\left(-\frac{1}{2}|E|, \frac{1}{2}|E|\right)
$$

be the interval centered about 0 for which $|E|=\left|E^{*}\right|$, where $|\cdot|$ is Lebesgue measure on $[-\pi, \pi]$. For $f \in L^{1}[-\pi, \pi]$ with $f \geqslant 0$, define

$$
f^{*}(x)=\int_{0}^{\infty} \chi_{\{f>t\}^{*}}(x) d t, \quad x \in[-\pi, \pi] .
$$

This function $f^{*}$ satisfies $f^{*}(x)=f^{*}(|x|)$ on $[-\pi, \pi]$ (i.e., symmetric), is non-increasing on $[0, \pi]$, and has the same integral as $f$. The important fact used here is the following [10, Ch. 10]. 
Lemma 6.9 (Hardy-Littlewood). For nonnegative and measurable $f, g$ we have

$$
\int_{-\pi}^{\pi} f(x) g(x) d x \leqslant \int_{-\pi}^{\pi} f^{*}(x) g^{*}(x) d x
$$

If $f$ is positive and symmetric on $[-\pi, \pi]$ and $f$ is decreasing on $[0, \pi]$, then for each $t$ the set $\{f>t\}$ is the interval

$$
\left(-\frac{1}{2}|\{f>t\}|, \frac{1}{2}|\{f>t\}|\right) \text {. }
$$

In other words, $\{f>t\}^{*}=\{f>t\}$. By the layer cake representation of $f$ we have

$$
\begin{aligned}
f(x) & =\int_{0}^{\infty} \chi_{\{f>t\}}(x) d t \\
& =\int_{0}^{\infty} \chi_{\{f>t\}^{*}}(x) d t \\
& =f^{*}(x) .
\end{aligned}
$$

Conclusion: If $f$ is positive, symmetric $(f(t)=f(|t|)$ on $[-\pi, \pi])$, and decreasing on $[0, \pi]$, then $f^{*}=f$ (almost everywhere).

Proof of Theorem 6.1. If $\varphi$ is outer and $\varphi\left(\lambda_{n}\right)=w_{n}$ for all $n \geqslant 1$, then $\psi=\varphi / M$ is outer and interpolates $w_{n} / M$. For $0<r<1$ we have

$$
\begin{aligned}
-(1-r) \log |\psi(r)|= & -(1-r) \int_{-\pi}^{\pi} P_{r}(t) \log \left|\psi\left(e^{i t}\right)\right| \frac{d t}{2 \pi} \\
= & -(1-r) \int_{-\pi}^{\pi} P_{r}(t) \max \left(0, \log \left|\psi\left(e^{i t}\right)\right|\right) \frac{d t}{2 \pi} \\
& -(1-r) \int_{-\pi}^{\pi} P_{r}(t) \min \left(0, \log \left|\psi\left(e^{i t}\right)\right|\right) \frac{d t}{2 \pi} \\
\leqslant & (1-r) \int_{-\pi}^{\pi} P_{r}(t) k(t) \frac{d t}{2 \pi},
\end{aligned}
$$

where $k=-\min (0, \log |\psi|)$ is nonnegative and integrable on $[-\pi, \pi]$.

Apply the Hardy-Littlewood estimate (Lemma 6.9) to (6.4) with $f=k$ and $g=P_{r}$ (which is already symmetric and so $g=g^{*}$ - see the discussion above) to obtain the estimate

$$
(1-r) \int_{-\pi}^{\pi} P_{r}(t) k(t) \frac{d t}{2 \pi} \leqslant(1-r) \int_{-\pi}^{\pi} P_{r}(t) k^{*}(t) \frac{d t}{2 \pi} .
$$


By (6.8) (note the use of the fact that $k^{*}(|t|)=k^{*}(t)$ )

$$
-(1-r) \log |\psi(r)| \leqslant \frac{2+\pi}{\pi} \int_{0}^{1-r} k^{*}(t) d t .
$$

Insert $r=\lambda_{n}$ into the above inequality to complete the proof.

Next we improve Theorem 6.1 with this sharpness result.

Theorem 6.10. Suppose $\left\{\lambda_{n}\right\} \subseteq(0,1)$ is interpolating and $h$ is a positive, decreasing, integrable function on $[0,1]$. If $\left\{w_{n}\right\} \subseteq \mathbb{C} \backslash\{0\}$ is bounded and satisfies

$$
-\left(1-\lambda_{n}\right) \log \left|w_{n}\right| \asymp \int_{0}^{1-\lambda_{n}} h(t) d t,
$$

then there is a bounded outer function $\psi$ such that

$$
-\left(1-\lambda_{n}\right) \log \psi\left(\lambda_{n}\right) \asymp \int_{0}^{1-\lambda_{n}} h(t) d t .
$$

In the statement above, $A_{n} \asymp B_{n}$ means there are positive constants $c_{1}$ and $c_{2}$, independent of $n$, such that $c_{1} A_{n} \leqslant B_{n} \leqslant c_{2} A_{n}$ for all $n$.

Proof. For $0<r<1$, (6.8) yields

$$
A_{h}(r) \asymp \int_{0}^{1-r} h(t) d t .
$$

If $\varphi$ is the (bounded) outer function with

$$
\left|\varphi\left(e^{i t}\right)\right|=e^{-h(|t|)}
$$

for almost every $t \in[-\pi, \pi]$ (see (2.2)), then

$$
\begin{aligned}
-(1-r) \log |\varphi(r)| & =-(1-r) \int_{-\pi}^{\pi} P_{r}(t) \log \left|\varphi\left(e^{i t}\right)\right| \frac{d t}{2 \pi} \\
& =A_{h}(r) \asymp \int_{0}^{1-r} h(t) d t .
\end{aligned}
$$

With $r=\lambda_{n}$ we have

$$
-\left(1-\lambda_{n}\right) \log \left|\varphi\left(\lambda_{n}\right)\right| \asymp \int_{0}^{1-\lambda_{n}} h(t) d t .
$$

Now apply Proposition 3.6 to produce a bounded outer function $\psi$ with $\psi\left(\lambda_{n}\right)=\left|\varphi\left(\lambda_{n}\right)\right|$. 


\section{More DELiCATE INTERPolation}

Given $h$ as in Theorem 6.10, there is a bounded outer $\varphi$ such that

$$
\log \varphi\left(\lambda_{n}\right) \asymp \frac{1}{1-\lambda_{n}} \int_{0}^{1-\lambda_{n}} h(t) d t, \quad n \geqslant 1 .
$$

Can we replace $\asymp$ with $=$ in the above? Equivalently, can we find an outer (bounded outer) $\varphi$ such that

$$
\varphi\left(\lambda_{n}\right)=\exp \left(-\frac{1}{1-\lambda_{n}} \int_{0}^{1-\lambda_{n}} h(t) d t\right), \quad n \geqslant 1 ?
$$

We certainly can find

$$
d_{n} \in\left[\frac{1}{2 \pi}, \frac{2+\pi}{\pi}\right]
$$

such that

$$
\varphi\left(\lambda_{n}\right)^{d_{n}}=\exp \left(-\frac{1}{1-\lambda_{n}} \int_{0}^{1-\lambda_{n}} h(t) d t\right) .
$$

By Theorem 3.1 and Remark 3.4 there is a bounded outer $\psi$ with $\Re \psi>0$ such that $\psi\left(\lambda_{n}\right)=1 / d_{n}$ for all $n$. The function $f=\varphi^{\psi}$ is analytic on $\mathbb{D}$ with

$$
f\left(\lambda_{n}\right)=\exp \left(-\frac{1}{1-\lambda_{n}} \int_{0}^{1-\lambda_{n}} h(t) d t\right)
$$

and thus performs the interpolation. But of course we need to check that $f$ is outer (bounded outer).

Indeed this is something that needs checking since if $\varphi$ and $\psi$ are outer, $f=\varphi^{\psi}$ need not be outer. In fact with $\varphi=e$ (constant outer function) and

$$
\psi(z)=-\frac{1+z}{1-z}
$$

then

$$
f=\varphi^{\psi}=\exp \left(-\frac{1+z}{1-z}\right)
$$

is inner! Here is our result concerning when $\varphi^{\psi}$ is outer (bounded outer).

Proposition 7.1. Let $\varphi$ be outer and $\psi$ be bounded and outer.

(i) If $\arg \varphi(\xi) \in L^{1}(\mathbb{T})$, then $f=\varphi^{\psi}$ is outer.

(ii) If $\Re \psi>0$ and $\arg \varphi(\xi) \in L^{\infty}(\mathbb{T})$, then $f=\varphi^{\psi}$ is outer and bounded. 
The proof of this proposition needs a few preliminaries. If $u \in L^{1}(\mathbb{T})$ and $u \geqslant 0$, the Herglotz integral

$$
H_{u}(z)=\int_{\mathbb{T}} \frac{\xi+z}{\xi-z} u(\xi) d m(\xi)
$$

is analytic on $\mathbb{D}$ and

$$
\Re H_{u}(z)=\int_{\mathbb{T}} \frac{1-|z|^{2}}{|\xi-z|^{2}} u(\xi) d m(\xi)>0, \quad z \in \mathbb{D} .
$$

By a known result [9, p. 65], $H_{u}$ is outer. Recall from \$2 the Hardy space $H^{1}$ and the Smirnov class $N^{+}$.

Lemma 7.3. For $f \in H^{1}$ there are $G_{j} \in N^{+}$with $\Re G_{j} \geqslant 0$ on $\mathbb{D}$ for $j=1,2$ such that $f=G_{1}-G_{2}$.

Proof. Functions in $H^{1}$ have radial boundary values almost everywhere on $\mathbb{T}$ and so let $u_{+}$and $u_{-}$be defined for almost every $\xi \in \mathbb{T}$ by

$$
u_{+}(\xi)=\max (\Re f(\xi), 0), \quad u_{-}(\xi)=\max (-\Re f(\xi), 0) .
$$

Since $|\Re f(\xi)| \leqslant|f(\xi)|$ and $|f|$ is integrable on $\mathbb{T}$, we see that $u_{+}, u_{-}$ are nonnegative integrable functions. Furthermore, by the discussion above, $H_{u_{+}}$and $H_{u_{-}}$belong to $N^{+}$and have positive real parts on $\mathbb{D}$. Finally, $H_{u_{+}}-H_{u_{-}}$belongs to $N^{+}$and has the same real part as $f$ on $\mathbb{T}$. Thus, by the uniqueness of the harmonic conjugate, $f=H_{u_{+}}-H_{u_{-}}+i c$ for some $c \in \mathbb{R}$. This completes the proof.

Lemma 7.4. If $f \in H^{1}$, then $e^{f}$ is outer.

Proof. By the previous lemma, $f=H_{u_{+}}-H_{u_{-}}+i c$ and so

$$
e^{f}=e^{i c} \frac{e^{-H_{u_{-}}}}{e^{-H_{u_{+}}}}
$$

From the formula for the Herglotz integral in (7.2) and the definition of outer from (2.1), the functions $e^{-H_{u_{+}}}$and $e^{-H_{u_{-}}}$are outer. Thus, $e^{f}$ is also outer.

Proof of Proposition 7.1. On $\mathbb{T}$ we have

$$
\begin{aligned}
|\log \varphi| & \leqslant|\log | \varphi||+|\arg \varphi| \\
& =\left|\log \left(|\varphi| /\|\varphi\|_{\infty}\right)+\log \|\varphi\|_{\infty}\right|+|\arg \varphi| \\
& \leqslant\left|\log \left(|\varphi| /\|\varphi\|_{\infty}\right)\right|+\left|\log \|\varphi\|_{\infty}\right|+|\arg \varphi| \\
& =-\log \left(|\varphi| /\|\varphi\|_{\infty}\right)+\left|\log \|\varphi\|_{\infty}\right|+|\arg \varphi|
\end{aligned}
$$




$$
\leqslant-\log |\varphi|+2 \log ^{+}\|\varphi\|_{\infty}+|\arg \varphi| \text {. }
$$

From $\log |\varphi| \in L^{1}(\mathbb{T})$ and $|\arg \varphi| \in L^{1}(\mathbb{T})$, follows $|\log \varphi| \in L^{1}(\mathbb{T})$. Since $\varphi$ is outer, $\log \varphi \in N^{+}$. A standard result [4, p. 28] of Smirnov implies $\log \varphi \in H^{1}$. Therefore, $\psi \log \varphi \in H^{1}$. By the previous lemma, $f=\exp (\psi \log \varphi)$ is outer. This proves (i).

If we assume that

$$
|\Im \log \varphi|=|\arg \varphi| \leqslant M \text { and } \Re \psi \geqslant 0
$$

on $\mathbb{T}$, we have

$$
\begin{aligned}
|f| & =\exp (\Re \psi \log |\varphi|-\Im \psi \arg \varphi) \\
& \leqslant \exp \left(\|\psi\|_{\infty} \log \left(1+\|\varphi\|_{\infty}\right)+M\|\psi\|_{\infty}\right) .
\end{aligned}
$$

Thus, $f$ is a bounded outer function. Note that

$$
\Re \psi \log |\varphi| \leqslant\|\psi\|_{\infty} \log \left(1+\|\varphi\|_{\infty}\right)
$$

follows from the fact that $\Re \psi \geqslant 0$ on $\mathbb{T}$. This proves (ii).

Let us use the results above to refine Theorem 6.10.

Theorem 7.5. Suppose $h$ is a positive, decreasing, integrable function on $[0,1]$. Let $\left\{\lambda_{n}\right\} \subseteq(0,1)$ be interpolating and $\left\{w_{n}\right\} \subseteq \mathbb{C} \backslash\{0\}$ be bounded with

$$
-\left(1-\lambda_{n}\right) \log \left|w_{n}\right| \asymp \int_{0}^{1-\lambda_{n}} h(t) d t, \quad n \geqslant 1 .
$$

(i) If $h(|t|) \log ^{+} h(|t|) \in L^{1}[-\pi, \pi]$ then there is an outer $\varphi$ such that $\varphi\left(\lambda_{n}\right)=w_{n}$ for all $n$.

(ii) If

$$
\mathrm{PV} \int_{-\pi}^{\pi} \cot \left(\frac{\theta-t}{2}\right) h(|t|) \frac{d t}{2 \pi}
$$

is bounded on $[-\pi, \pi]$ then there is a bounded outer $\varphi$ such that $\varphi\left(\lambda_{n}\right)=w_{n}$ for all $n$.

Proof. From the discussion at the very beginning of this section, we can find bounded outer $\varphi$ and $\psi$ such that $f=\varphi^{\psi}$ satisfies $f\left(\lambda_{n}\right)=w_{n}$ for all $n$. We just need to check that $f$ is outer (bounded outer).

By the proof of Theorem 6.10 and (6.11), $\log \left|\varphi\left(e^{i t}\right)\right|=-h(|t|)$ and

$$
\varphi(z)=\exp \left(\int_{\mathbb{T}} \frac{\xi+z}{\xi-z} \log |\varphi(\xi)| d m\right)
$$




$$
=\exp \left(\int_{\mathbb{T}} \Re\left(\frac{\xi+z}{\xi-z}\right) \log |\varphi(\xi)| d m+i \int_{\mathbb{T}} \Im\left(\frac{\xi+z}{\xi-z}\right) \log |\varphi(\xi)| d m\right) .
$$

From

$$
\arg \varphi(z)=i \int_{\mathbb{T}} \Im\left(\frac{\xi+z}{\xi-z}\right) \log |\varphi(\xi)| d m, \quad z \in \mathbb{D},
$$

and standard theory involving the Hilbert transform on the circle we have

$$
\arg \varphi\left(e^{i \theta}\right)=-\mathrm{PV} \int_{-\pi}^{\pi} \cot \left(\frac{\theta-t}{2}\right) h(|t|) \frac{d t}{2 \pi} .
$$

A classical result of Zygmund [15, Vol I, p. 254] says that if the function $h(|t|) \log ^{+} h(|t|)$ belongs to $L^{1}[-\pi, \pi]$ then $\arg \varphi \in L^{1}(\mathbb{T})$. An application of Proposition 7.1 yields $f=\varphi^{\psi}$ is outer.

If the above Hilbert transform is bounded, another application of Proposition 17.1, along with the fact that we can always choose $\psi$ so that $\Re \psi>0$ (Remark 3.4), yields $f=\varphi^{\psi}$ is bounded and outer.

Example 7.6. If $\left\{\lambda_{n}\right\} \subseteq(0,1)$ is interpolating, we know from Theorem 4.1 that any $\varphi \in N^{+}$with

$$
\varphi\left(\lambda_{n}\right)=\exp \left(-\frac{2}{1-\lambda_{n}}\right), \quad n \geqslant 1
$$

must have an inner factor. In fact, the obvious guess at an analytic functions that interpolates this sequence is

$$
\varphi(z)=\exp \left(-\frac{2}{1-z}\right)
$$

turns out to be a constant multiple of an inner function. Indeed, the singular inner function

$$
\exp \left(-\frac{1+z}{1-z}\right)
$$

can be written as

$$
\begin{aligned}
\exp \left(-\frac{1+z}{1-z}\right) & =\exp \left(-\frac{2-(1-z)}{1-z}\right) \\
& =\exp \left(-\frac{2}{1-z}\right) e .
\end{aligned}
$$

Thus $\varphi$ is a constant multiple of a singular inner function.

Example 7.7. Let

$$
w_{n}=\exp \left(-\frac{1}{1-\lambda_{n}} \frac{1}{\left(\log \frac{100}{1-\lambda_{n}}\right)^{2}}\right) .
$$


Here

$$
h(t)=\frac{2}{t\left(\log \left(\frac{100}{t}\right)\right)^{3}}, \quad 0<t<1,
$$

is $h$ is positive and decreasing on $[0,1]$ and $h(|t|) \log ^{+} h(|t|)$ belongs to $L^{1}[-1,1]$. Thus $\left\{w_{n}\right\}$ can be interpolated with an outer function.

Example 7.8. Let

$$
w_{n}=\exp \left(-\frac{1}{\left(1-\lambda_{n}\right)^{\alpha}}\right)
$$

where $0<\alpha<1$. In this case,

$$
h(t)=\frac{1-\alpha}{t^{\alpha}}
$$

is positive, decreasing, and $h(|t|) \log ^{+} h(|t|) \in L^{1}[-\pi, \pi]$. Thus, by the previous theorem, $\left\{w_{n}\right\}$ can be interpolated by an outer function. In fact, one can take $\varphi$ to be a bounded outer function. To see this, observe that $(1-z)^{-\alpha} \in H^{1}$ and so

$$
\varphi(z)=\exp \left(-\frac{1}{(1-z)^{\alpha}}\right)
$$

is outer (Lemma 7.4). Furthermore,

$$
\begin{aligned}
\frac{1}{1-e^{i \theta}} & =\frac{e^{i \theta / 2}}{e^{-i \theta / 2}-e^{i \theta / 2}} \\
& =\frac{e^{-i \theta / 2}}{-2 i \sin (\theta / 2)} \\
& =\frac{1}{2 \sin (\theta / 2)} e^{i \frac{\pi-\theta}{2}} .
\end{aligned}
$$

Thus,

$$
\left|\varphi\left(e^{i \theta}\right)\right| \leqslant e^{-2^{-\alpha} \cos (\pi \alpha / 2)}, \quad \theta \in[-\pi, \pi],
$$

and so $\varphi$ is outer and $\varphi$ is bounded on $\mathbb{T}$. A result of Smirnov [4, p. 28] says that $\varphi \in H^{\infty}$. If $0<m \leqslant d_{n} \leqslant M<\infty$, one can also interpolate

$$
w_{n}=\exp \left(-d_{n} \frac{1}{\left(1-\lambda_{n}\right)^{\alpha}}\right)
$$

with an outer function.

Example 7.9. If $\left\{\lambda_{n}\right\} \subseteq(0,1)$ is interpolating and $\left\{d_{n}\right\}$ satisfies $0<$ $m \leqslant d_{n} \leqslant M<\infty$ for all $n \geqslant 1$, one can appeal to Proposition [7.1] directly to interpolate

$$
w_{n}=\left(1-\lambda_{n}\right)^{d_{n}}
$$


with a bounded outer function. Here $f=\varphi^{\psi}$, where $\varphi(z)=1-z$ (which clearly has bounded argument) and $\psi$ is the bounded outer function with $\Re \psi>0$ and $\psi\left(\lambda_{n}\right)=d_{n}$ for all $n \geqslant 1$.

\section{COMmon RAnge}

In this section, we present an application of our outer interpolation results. For $\varphi \in H^{\infty}$ let $T_{\bar{\varphi}}$ denote the co-analytic Toeplitz operator on the Hardy space $H^{2}$. By this we mean the operator $T_{\bar{\varphi}}: H^{2} \rightarrow$ $H^{2}$ defined by $T_{\bar{\varphi}} f=P_{+}(\bar{\varphi} f)$, where $P_{+}$is the standard orthogonal projection of $L^{2}(\mathbb{T})$ onto $H^{2}$. See [8, Ch. 4] for the basics of Toeplitz operators. Let

$$
\mathscr{R}\left(H^{2}\right):=\bigcap\left\{T_{\bar{\varphi}} H^{2}: \varphi \in H^{\infty} \backslash\{0\}\right\}
$$

denote the common range of the (nonzero) co-analytic Toeplitz operators on $H^{2}$. A well-known result is the following:

Theorem 8.1 (McCarthy [12]).

$$
\mathscr{R}\left(H^{2}\right)=\left\{f \in H^{\infty}: \widehat{f}(n)=O\left(e^{-c_{f} \sqrt{n}}\right)\right\} .
$$

The above decay on the Fourier coefficients $\widehat{f}(n)$ shows that $\left\{n^{K} \widehat{f}(n)\right\}$ is absolutely summable for all $K \geqslant 0$ and so functions in $\mathscr{R}\left(H^{2}\right)$ must be infinitely differentiable on $\overline{\mathbb{D}}$.

The Douglas factorization theorem [3] implies that $T_{\bar{\varphi}} H^{2}=T_{\overline{\varphi_{0}}} H^{2}$, where $\varphi_{0}$ is the outer part of $\varphi \in H^{\infty}$. Thus,

$$
\mathscr{R}\left(H^{2}\right)=\bigcap\left\{T_{\bar{\varphi}} H^{2}: \varphi \in H^{\infty} \cap \mathcal{O}\right\} .
$$

Recall that $\mathcal{O}$ are the outer functions. Also important here is that $T_{\bar{\varphi}}$ is injective whenever $\varphi \in H^{\infty} \cap \mathcal{O}$.

What does this common range problem look like in model spaces? For an inner function $u$, the model space $\mathcal{K}_{u}:=\left(u H^{2}\right)^{\perp}$ is an invariant subspace for any co-analytic Toeplitz operator $T_{\bar{\varphi}}, \varphi \in H^{\infty}$. For this and other facts about model spaces used in this section, we refer the reader to [8]. For a fixed inner function $u$, what is

$$
\mathscr{R}\left(\mathcal{K}_{u}\right):=\bigcap\left\{T_{\bar{\varphi}} \mathcal{K}_{u}: \varphi \in H^{\infty} \cap \mathcal{O}\right\} ?
$$

Since $T_{\bar{\varphi}} \mathcal{K}_{u} \subseteq T_{\bar{\varphi}} H^{2}$ we have $\mathscr{R}\left(\mathcal{K}_{u}\right) \subseteq \mathscr{R}\left(H^{2}\right)$ but the inclusion can be strict (see below). Furthermore, $\mathscr{R}\left(\mathcal{K}_{u}\right) \subseteq \mathcal{K}_{u}$ since $T_{\bar{\varphi}} \mathcal{K}_{u} \subseteq \mathcal{K}_{u}$ for all bounded outer $\varphi$. 
Example 8.2. If $u(z)=z^{N}$ then $\mathcal{K}_{u}=\mathscr{P}_{N-1}$, the polynomials of degree at most $N-1$. Since $\varphi$ is outer, $T_{\bar{\varphi}}$ is injective and so $T_{\bar{\varphi}} \mathscr{P}_{N-1}=$ $\mathscr{P}_{N-1}$. So in this case $\mathscr{R}\left(\mathcal{K}_{u}\right)=\mathscr{P}_{N-1}$.

Example 8.3. In a similar way, for a finite Blaschke product $u$ with distinct zeros $\lambda_{1}, \ldots, \lambda_{n}$ in $\mathbb{D}$, we have $\mathcal{K}_{u}=\bigvee\left\{k_{\lambda_{1}}, \ldots, k_{\lambda_{n}}\right\}$, where $k_{\lambda}(z)=\frac{1}{1-\bar{\lambda} z}$ are the Cauchy kernels for $H^{2}$. It follows, using

$$
T_{\bar{\varphi}} k_{\lambda_{j}}=\overline{\varphi\left(\lambda_{j}\right)} k_{\lambda_{j}}
$$

and $\varphi\left(\lambda_{j}\right) \neq 0$, that $\mathscr{R}\left(\mathcal{K}_{u}\right)=\bigvee\left\{k_{\lambda_{1}}, \ldots, k_{\lambda_{n}}\right\}$.

What are some inhabitants of $\mathscr{R}\left(\mathcal{K}_{u}\right)$ when $u$ is not finite a Blaschke product? If $\lambda$ is a zero of $u$ then $k_{\lambda} \in \mathcal{K}_{u}$ and so $k_{\lambda} \in \mathscr{R}\left(\mathcal{K}_{u}\right)$ as argued in Example 8.3. It is more difficult to identify other elements of $\mathscr{R}\left(\mathcal{K}_{u}\right)$.

Remark 8.5. Since $\mathscr{R}\left(\mathcal{K}_{u}\right) \subseteq \mathcal{K}_{u}$, then $\mathscr{R}\left(\mathcal{K}_{u}\right)$ will inherit the properties of functions in $\mathcal{K}_{u}$. For example, if

$$
\left\{\xi \in \mathbb{T}: \underline{\lim _{z \rightarrow \xi}}|u(z)|=0\right\}
$$

the boundary spectrum of $u$, omits an an arc $I$ of $\mathbb{T}$, then function in $\mathcal{K}_{u}$ will have an analytic continuation across $I$. Hence functions $\mathscr{R}\left(\mathcal{K}_{u}\right)$ will also have this property.

Example 8.6. It is possible to produce a suitable singular inner function $u$ for which $\mathcal{K}_{u}$ contains no nonzero smooth functions [5]. Since $\mathscr{R}\left(\mathcal{K}_{u}\right)$ is contained in the smooth functions (Theorem 1 .11), it follows that $\mathscr{R}\left(\mathcal{K}_{u}\right)=\{0\}$.

Theorem 8.7. If $u$ is inner then

$$
\mathscr{R}\left(\mathcal{K}_{u}\right)=\mathcal{K}_{u} \cap \mathscr{R}\left(H^{2}\right)=\left\{f \in \mathcal{K}_{u}: \widehat{f}(n)=O\left(e^{-c_{f} \sqrt{n}}\right)\right\} .
$$

Proof. The containment $\subseteq$ is automatic. Now suppose $f \in \mathcal{K}_{u} \cap \mathscr{R}\left(H^{2}\right)$. Given any $\varphi \in H^{\infty} \cap \mathcal{O}$ there is a $g_{\varphi} \in H^{2}$ for which $f=T_{\bar{\varphi}} g_{\varphi}$. Since $f \in \mathcal{K}_{u}$ we have $\langle f, u h\rangle=0$ for all $h \in H^{2}$. Using the fact that $T_{\bar{\varphi}}^{*}=T_{\varphi}$ (which is just multiplication by $\varphi$ ), this implies

$$
0=\langle f, u h\rangle=\left\langle T_{\bar{\varphi}} g_{\varphi}, u h\right\rangle=\left\langle g_{\varphi}, \varphi u h\right\rangle, \quad h \in H^{2} .
$$

The function $\varphi$ is outer and so $\left\{\varphi h: h \in H^{2}\right\}$ is dense in $H^{2}$ (Beurling's theorem [4, p. 114]). Thus $\left\langle g_{\varphi}, u k\right\rangle=0$ for all $k \in H^{2}$ and so $g_{\varphi} \in \mathcal{K}_{u}$. Thus, $f \in \mathscr{R}\left(\mathcal{K}_{u}\right)$. 
Though Theorem 8.7 is a description of $\mathscr{R}\left(\mathcal{K}_{u}\right)$, it can be difficult to apply. Indeed, the precise contents of a model space are not always well understood and thus determining which of them have the right smoothness property can be quite challenging. In the next section we focus on a special class of inner functions $u$ where we better understand $\mathcal{K}_{u}$ as well as $\mathscr{R}\left(\mathcal{K}_{u}\right)$.

\section{Interpolating Blaschke products}

In Example 8.3 we computed $\mathscr{R}\left(\mathcal{K}_{B}\right)$ when $B$ is a finite Blaschke product. In this section we extend our discussion to interpolating Blaschke products. Let

$$
\kappa_{\lambda}:=\frac{k_{\lambda}}{\left\|k_{\lambda}\right\|}=\frac{\sqrt{1-|\lambda|^{2}}}{1-\bar{\lambda} z}, \quad \lambda \in \mathbb{D},
$$

denote the normalized Cauchy kernel for $H^{2}$. This next proposition is a well-known fact about model spaces [8, p. 277].

Proposition 9.1. If $B$ is an interpolating Blaschke product with zeros $\left\{\lambda_{n}\right\}$, then $\left\{\kappa_{\lambda_{n}}\right\}$ is a Riesz basis for $\mathcal{K}_{B}$. Hence each $f \in \mathcal{K}_{B}$ has a unique representation as $f=\sum_{n \geqslant 1} a_{n} \kappa_{\lambda_{n}}$, where $\left\{a_{n}\right\} \in \ell^{2}$, that is, $\sum_{\mathcal{K}_{B} \geqslant 1}\left|a_{n}\right|^{2}<\infty$. Conversely, any such linear combination belongs to

We now obtain a more tangible description of $\mathscr{R}\left(\mathcal{K}_{B}\right)$ than the one in Theorem 8.7. We start with the following lemma.

Lemma 9.2. Let $\varphi$ be bounded and outer and $B$ be an interpolating Blaschke product with zeros $\left\{\lambda_{n}\right\}$. Then

$$
T_{\bar{\varphi}} \mathcal{K}_{B}=\left\{\sum_{n=1}^{\infty} b_{n} \kappa_{\lambda_{n}}: \sum_{n=1}^{\infty} \frac{\left|b_{n}\right|^{2}}{\left|\varphi\left(\lambda_{n}\right)\right|^{2}}<\infty\right\} .
$$

Proof. Suppose

$$
\sum_{n=1}^{\infty} \frac{\left|b_{n}\right|^{2}}{\left|\varphi\left(\lambda_{n}\right)\right|^{2}}<\infty
$$

Then

$$
f=\sum_{n=1}^{\infty} \frac{b_{n}}{\overline{\varphi\left(\lambda_{n}\right)}} \kappa_{\lambda_{n}} \in \mathcal{K}_{B}
$$


(Proposition 9.1) and, by (8.4),

$$
T_{\bar{\varphi}} f=\sum_{n=1}^{\infty} \frac{b_{n}}{\overline{\varphi\left(\lambda_{n}\right)}} \overline{\varphi\left(\lambda_{n}\right)} \kappa_{\lambda_{n}}=\sum_{n=1}^{\infty} b_{n} \kappa_{\lambda_{n}} .
$$

Thus, $\sum_{n \geqslant 1} b_{n} \kappa_{\lambda_{n}} \in T_{\bar{\varphi}} \mathcal{K}_{B}$.

Conversely, suppose $g=T_{\bar{\varphi}} f$ for some $f \in \mathcal{K}_{B}$. Then $f=\sum_{n \geqslant 1} a_{n} \kappa_{\lambda_{n}}$ for some unique $\left\{a_{n}\right\} \in \ell^{2}$. From Proposition 9.1

$$
g=T_{\bar{\varphi}} f=\sum_{n=1}^{\infty} a_{n} \overline{\varphi\left(\lambda_{n}\right)} \kappa_{\lambda_{n}}
$$

and so $\left\{a_{n} \overline{\varphi\left(\lambda_{n}\right)}\right\}=\left\{b_{n}\right\} \in \ell^{2}$. Thus, $\left\{a_{n}\right\}=\left\{b_{n} \overline{\varphi\left(\lambda_{n}\right)}\right\} \in \ell^{2}$.

Here is our description of $\mathscr{R}\left(\mathcal{K}_{B}\right)$.

Theorem 9.4. Suppose $B$ is an interpolating Blaschke product with zeros $\left\{\lambda_{n}\right\} \subseteq(0,1)$. For $\left\{a_{n}\right\} \in \ell^{2}$ and $f=\sum_{n \geqslant 1} a_{n} \kappa_{\lambda_{n}}$, the following are equivalent:

(i) $f \in \mathscr{R}\left(\mathcal{K}_{B}\right)$;

(ii) The sum

$$
\sum_{n=1}^{\infty} \frac{\left|a_{n}\right|^{2}}{\left|\varphi\left(\lambda_{n}\right)\right|}
$$

is finite for every bounded outer function $\varphi$.

(iii) The sum

$$
\sum_{n=1}^{\infty}\left|a_{n}\right|^{2} \exp \left(\frac{1}{1-\lambda_{n}} \int_{0}^{1-\lambda_{n}} h(t) d t\right)
$$

is finite for every positive, decreasing, integrable function $h$ on $[0,1]$.

(iv)

$$
\sum_{n=1}^{\infty} a_{n} \sqrt{1-\left|\lambda_{n}\right|^{2}} \lambda_{n}^{N}=O\left(e^{-c_{f} \sqrt{N}}\right), \quad N \rightarrow \infty .
$$

Proof. The proof of (i) $\Longleftrightarrow$ (ii) follows from Lemma 9.2 . The proof of (ii) $\Longleftrightarrow$ (iii) follows from Theorem 6.10. For the proof that (i) $\Longleftrightarrow$ (iv), note that

$$
f^{(N)}(z)=N ! \sum_{n=1}^{\infty} a_{n} \sqrt{1-\left|\lambda_{n}\right|^{2}} \frac{\lambda_{n}^{N}}{\left(1-\lambda_{n} z\right)^{N+1}}
$$


and thus

$$
\widehat{f}(N)=\frac{f^{(N)}(0)}{N !}=\sum_{n=1}^{\infty} a_{n} \sqrt{1-\left|\lambda_{n}\right|^{2}} \lambda_{n}^{N} .
$$

Now apply Theorem 8.7 .

To obtain a rich class of functions in $\mathscr{R}\left(\mathcal{K}_{B}\right)$, besides the obvious finite linear combinations of $\kappa_{\lambda_{n}}$, Lemma 4.3 says that

$$
-\left(1-\lambda_{n}\right) \log \left|\varphi\left(\lambda_{n}\right)\right| \rightarrow 0, \quad \varphi \in H^{\infty} \cap \mathcal{O} .
$$

If $c>0$ and $\left\{a_{n}\right\}$ satisfies

$$
\sum_{n=1}^{\infty}\left|a_{n}\right|^{2} \exp \left(\frac{c}{1-\lambda_{n}}\right)<\infty
$$

then

$$
\frac{1}{\left|\varphi\left(\lambda_{n}\right)\right|} \leqslant \exp \left(\frac{c}{1-\lambda_{n}}\right)
$$

for sufficiently large enough $n$. Thus, $f=\sum_{n \geqslant 1} a_{n} \kappa_{\lambda_{n}} \in \mathscr{R}\left(\mathcal{K}_{B}\right)$. In other words,

$$
\bigcup_{c>0}\left\{\sum_{n=1}^{\infty} a_{n} \kappa_{\lambda_{n}}: \sum_{n=1}^{\infty}\left|a_{n}\right|^{2} \exp \left(\frac{c}{1-\lambda_{n}}\right)<\infty\right\} \subseteq \mathscr{R}_{B} .
$$

\section{Correct Definition of the Common Range?}

When $\varphi \in H^{\infty} \backslash\{0\}$ it is easy to show that $T_{\bar{\varphi}} H^{2}$ is dense in $H^{2}$. Thus

$$
\bigcap\left\{T_{\bar{\varphi}} H^{2}: \varphi \in H^{\infty} \backslash\{0\}\right\}
$$

the common range of the non-zero co-analytic Toeplitz operators, is meaningful. It just so happens, through the Douglas factorization theorem mentioned earlier, that $T_{\bar{\varphi}} H^{2}=T_{\overline{\varphi_{0}}} H^{2}$, where $\varphi_{0}$ is the outer factor of $\varphi$ and thus

$$
\bigcap\left\{T_{\bar{\varphi}} H^{2}: \varphi \in H^{\infty} \backslash\{0\}\right\}=\bigcap\left\{T_{\bar{\varphi}} H^{2}: \varphi \in H^{\infty} \cap \mathcal{O}\right\} .
$$

It made sense to us to define the common range of the co-analytic Toeplitz operators in the model space $\mathcal{K}_{u}$ as

$$
\bigcap\left\{T_{\bar{\varphi}} \mathcal{K}_{u}: \varphi \in H^{\infty} \cap \mathcal{O}\right\} .
$$

Of course the intersection

$$
\bigcap\left\{T_{\bar{\varphi}} \mathcal{K}_{u}: \varphi \in H^{\infty} \backslash\{0\}\right\}=\{0\}
$$


since $\operatorname{Ker} T_{\bar{u}}=\mathcal{K}_{u}$ so there needs to be some further restriction on the intersection. One might wonder if the "correct" definition of the common range in the model space should be

$$
\bigcap\left\{T_{\bar{\varphi}} \mathcal{K}_{u}: \varphi \in \mathcal{F}\right\},
$$

where $\mathcal{F}$ is the set of $\varphi \in H^{\infty}$ such that $T_{\bar{\varphi}} \mathcal{K}_{u}$ is dense in $\mathcal{K}_{u}$. One could make a case for this definition. However, the resulting common range may not be all that interesting.

For example, if $B$ is an interpolating Blaschke product with zeros $\left\{\lambda_{n}\right\}$, the fact that $\left\{\kappa_{\lambda_{n}}\right\}$ is minimal, in fact uniformly minimal [ㅇ, p. 277], shows that $T_{\bar{\varphi}} \mathcal{K}_{B}$ is dense in $\mathcal{K}_{B}$ if and only if $\varphi\left(\lambda_{n}\right) \neq 0$ for all $n$. From (9.3) we have

$$
T_{\bar{\varphi}} \mathcal{K}_{B}=\left\{\sum_{n=1}^{\infty} b_{n} \kappa_{\lambda_{n}}: \sum_{n=1}^{\infty} \frac{\left|b_{n}\right|^{2}}{\left|\varphi\left(\lambda_{n}\right)\right|^{2}}<\infty\right\} .
$$

Thus if $\varphi \in H^{\infty}$ interpolates the nonzero values of $b_{n}$ (which can be done via Carleson's theorem), the quantity

$$
\sum_{n=1}^{\infty} \frac{\left|b_{n}\right|^{2}}{\left|\varphi\left(\lambda_{n}\right)\right|^{2}}
$$

will be infinite whenever there are an infinite number of nonzero $b_{n}$. Thus $\mathcal{F}$ will consist of the $\varphi \in H^{\infty}$ such that $\varphi\left(\lambda_{n}\right) \neq 0$ for all $n$ and

$$
\bigcap\left\{T_{\bar{\varphi}} \mathcal{K}_{B}: \varphi \in \mathcal{F}\right\}
$$

will just be the finite linear combinations of the $\kappa_{\lambda_{n}}$.

\section{REFERENCES}

[1] Jim Agler and John E. McCarthy. Pick interpolation and Hilbert function spaces, volume 44 of Graduate Studies in Mathematics. American Mathematical Society, Providence, RI, 2002.

[2] Lennart Carleson. An interpolation problem for bounded analytic functions. Amer. J. Math., 80:921-930, 1958.

[3] R. G. Douglas. On majorization, factorization, and range inclusion of operators on Hilbert space. Proc. Amer. Math. Soc., 17:413-415, 1966.

[4] P. L. Duren. Theory of $H^{p}$ spaces. Academic Press, New York, 1970.

[5] Konstantin Dyakonov and Dmitry Khavinson. Smooth functions in starinvariant subspaces. In Recent advances in operator-related function theory, volume 393 of Contemp. Math., pages 59-66. Amer. Math. Soc., Providence, RI, 2006.

[6] Konstantin Dyakonov and Artur Nicolau. Free interpolation by nonvanishing analytic functions. Trans. Amer. Math. Soc., 359(9):4449-4465, 2007.

[7] J. P. Earl. On the interpolation of bounded sequences by bounded functions. J. London Math. Soc. (2), 2:544-548, 1970. 
[8] Stephan Ramon Garcia, Javad Mashreghi, and William T. Ross. Introduction to model spaces and their operators, volume 148 of Cambridge Studies in Advanced Mathematics. Cambridge University Press, Cambridge, 2016.

[9] J. Garnett. Bounded Analytic Functions, volume 236 of Graduate Texts in Mathematics. Springer, New York, first edition, 2007.

[10] G. H. Hardy, J. E. Littlewood, and G. Pólya. Inequalities. Cambridge, at the University Press, 1952. 2d ed.

[11] Paul Koosis. Introduction to $H_{p}$ spaces, volume 115 of Cambridge Tracts in Mathematics. Cambridge University Press, Cambridge, second edition, 1998. With two appendices by V. P. Havin [Viktor Petrovich Khavin].

[12] John E. McCarthy. Common range of co-analytic Toeplitz operators. J. Amer. Math. Soc., 3(4):793-799, 1990.

[13] H. S. Shapiro and A. L. Shields. On some interpolation problems for analytic functions. Amer. J. Math., 83:513-532, 1961.

[14] Roman Wituła, Edyta Hetmaniok, and Damian Słota. A stronger version of the second mean value theorem for integrals. Comput. Math. Appl., 64(6):1612$1615,2012$.

[15] A. Zygmund. Trigonometric series. Vol. I, II. Cambridge Mathematical Library. Cambridge University Press, Cambridge, third edition, 2002. With a foreword by Robert A. Fefferman.

DÉpartement de mathématiques et de statistique, Université Laval, Québec, QC, Canada, G1K 0A6

Email address: javad.mashreghi@mat.ulaval.ca

Department of Applied Mathematics, University of Agriculture, ul. Balicka 253C, 30-198 Kraków, Poland.

Email address: rmptak@cyf-kr.edu.pl

Department of Mathematics and Computer Science, University of RichMOND, Richmond, VA 23173, USA

Email address: wross@richmond.edu 